\title{
Visually perceived location is an invariant in the control of action
}

\author{
JOHN W. PHILBECK, JACK M. LOOMIS, and ANDREW C. BEALL \\ University of California, Santa Barbara, California
}

\begin{abstract}
We provide experimental evidence that perceived location is an invariant in the control of action, by showing that different actions are directed toward a single visually specified location in space (corresponding to the putative perceived location) and that this single location, although specified by a fixed physical target, varies with the availability of information about the distance of that target. Observers in two conditions varying in the availability of egocentric distance cues viewed targets at 1.5, 3.1 , or $6.0 \mathrm{~m}$ and then attempted to walk to the target with eyes closed using one of three paths; the path was not specified until after vision was occluded. The observers stopped at about the same location regardless of the path taken, providing evidence that action was being controlled by some invariant, ostensibly visually perceived location. That it was indeed perceived location was indicated by the manipulation of information about target distance-the trajectories in the full-cues condition converged near the physical target locations, whereas those in the reduced-cues condition converged at locations consistent with the usual perceptual errors found when distance cues are impoverished.
\end{abstract}

Currently, there are two very different conceptions of how vision controls action. The first of these is identified with the ecological approach to vision (e.g., Gibson, 1979; Lee, 1980; Turvey \& Carello, 1986; Warren, 1990); its starting point is the assumption that the changing visual stimulation of a moving observer is sufficient to control the observer's action. Going further, this conception is that very specific aspects of this changing visual stimulation are tightly coupled to particular aspects of the action. In this view, understanding of the visual control of action is possible without an explicit consideration of the mechanisms intervening between stimulation and action. The top panel of Figure 1 depicts this idea of perception/action coupling. The seemingly endless variety of locomotor behaviors is in fact constrained in its dimensionality by the physics of actual motion and by the structural properties of the human body. Similarly, the high dimensionality of visual stimulation can be reduced by exploiting aspects of the stimulus that are sufficient for controlling particular actions. Thus, one might control walking to an object by activating muscle groups so that the image of the object remains centered on the fovea and increases in size during locomotion (Gibson, 1958). Similarly, braking can be controlled by the derivative of an optical variable relating to optical expansion (Yilmaz \& Warren, 1995), steering can

This research was supported by ONR Grant N00014-95-1-0573. These data were presented at the 1995 annual meeting of the Association for Research in Vision and Ophthalmology. A preliminary report of the experiment appeared in Loomis, Da Silva, Philbeck, and Fukusima (1996). The authors thank Reginald Golledge and Daniel Montello for the use of their laboratory. Correspondence concerning this article should be addressed to J. W. Philbeck or J. M. Loomis, Department of Psychology, University of California, Santa Barbara, CA 931069660 (e-mail: philbeck@psych.ucsb.edu; loomis@psych.ucsb.edu; beall@psych.ucsb.edu). be controlled by global radial outflow of point features or by optical splay of path markers (Beall \& Loomis, 1996; Calvert, 1954; Royden, Crowell, \& Banks, 1994; Warren \& Hannon, 1990), and ball catching can be controlled by moving so as to maintain a linear optical trajectory of the ball (McBeath, Shaffer, \& Kaiser, 1995). Specific optical variables that are sufficient for controlling particular actions are referred to as optical invariants, for they bear an invariant relation with the actions they control.

The other conception of how vision controls actions hypothesizes that internal representations, resulting from sensory, perceptual, and cognitive processing, play an essential role in the explanation of at least some actions. One such representation, in particular, is referred to as visual space or visually perceived space. It is the output of visual processing that we experience as the spatial layout of objects and surfaces existing independently of ourselves (see, e.g., Loomis, 1992; Loomis, Da Silva, Fujita, \& Fukusima, 1992). Much research over the years has been directed toward elucidating functional properties of visual space (e.g., its degree of correspondence with physical space) and toward understanding the sensory and perceptual processes underlying this representation (e.g., Baird, 1970; Cutting \& Vishton, 1995; Foley, 1977, 1980; Gogel, 1990, 1993; Norman, Todd, Perotti, \& Tittle, 1996).

What is meant by the statement that an internal representation plays an essential role in the control of action? It is that the simplest explanation of action employs one or more intervening variables that result from sensory, perceptual, and cognitive processing but are prior to the observable variables of action. We elucidate this in connection with the internal variable of "perceived location" as depicted in the bottom panel of Figure 1, fashioned after the "lens model" of Brunswik (1956; see also Campbell, 1966). In this visual space conception, visually perceived 

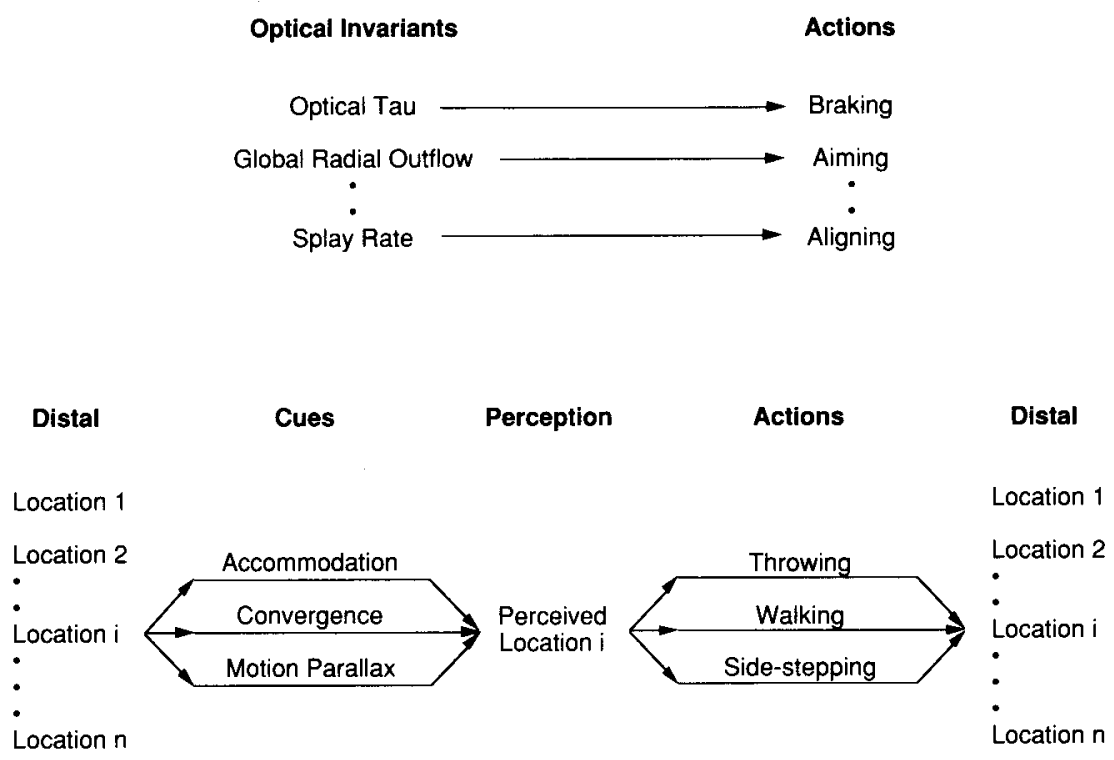

Figure 1. Top: the ecological conception of perception-action coupling, whereby different optical invariants in the visual stimulus control very specific actions; in this conception, perceived location plays no role in the explanation. Bottom: depiction of perceived location as an invariant in the control of action, in the spirit of Brunswik's "lens model" (1956). In the case depicted, a target is placed at location $i$. Various cues, along with internal constraints, codetermine the corresponding perceived location, toward which a variety of actions may be directed. Under full-cue conditions, the location toward which action is directed is coincident or nearly so with that of the target (as depicted here), but when cues are reduced, action may be directed to a location other than that of the physical target.

location is a three-dimensional internal variable that exists apart from any of the various stimulus cues that determine its value and apart from any single action that it participates in controlling. On the input side, multiple stimulus cues, along with internal constraints within the nervous system, serve to specify a particular location in visual space. Perceived location is not associated with any single cue, but is jointly determined by a multiplicity of cues and internal constraints; if we think of the cue values for a set of 10 cues as constituting a vector, different cue vectors might well specify the same perceived location (Gogel, 1984, 1990, 1993). For example, a change in the value of absolute motion parallax might compensate for a change in the value of binocular convergence, keeping perceived location constant.

The figure also conveys the idea that, on the output side, different actions can be controlled by the one internal variable, perceived location. Once the perceived location is specified, the observer can direct action to that location in a multitude of ways, even without obtaining additional sensory input during the action (throwing an object, blindly walking to it, etc.). In this view, perceived location is then an "invariant," "determining process," or "final common pathway" in the causal chain determining action. Different sensory cues are funneled through the internal variable of perceived location, and different actions are constrained by this variable. Besides Brunswik (1956; see also Campbell, 1966), Gogel (1993) and Foley (1977) have most fully articulated this idea (but in terms of perceived distance rather than perceived location). If one finds that different behaviors converge on a single location in space that is specified by a combination of sensory cues rather than by any one of them, the simplest explanation is that the different behaviors are being controlled by the internal variable of perceived location.

The experiment that we present here provides evidence that perceived location is an invariant in the control of action by showing that different actions are directed toward a single location in space (corresponding to the putative perceived location) and that this single location, although specified by a fixed physical target, varies with the availability of information about the distance of that target. The experiment employs a type of behavior variously referred to as visually directed action, open-loop responding, or locomotor pointing. In such a task, the observer views a target at some location and then, with eyes closed, carries out some action directed toward the location of the target. The most common action that has been used in human research of this kind is visually directed walking, in which the observer attempts to walk without vision to the location of the previously viewed target (Corlett, 1992; Elliott, 1986, 1987; Glasauer, Amorim, Vitte, \& Berthoz, 1994; Laurent \& Cavallo, 1985; Loomis et al., 1992; Rieser, Ashmead, Talor, \& Youngquist, 1990; Rieser, Pick, Ashmead, \& Garing, 1995; Steenhuis \& Goodale, 1988; Thomson, 1980, 1983). Other actions that have been used are visually directed reaching, in which the observer views a target within arm's reach and then 
attempts to point to the target without visual information about the hand (Foley, 1977), and visually directed throwing, in which the observer views a distant target and attempts to throw a ball or other object to its location without receiving visual feedback about the throw (Eby \& Loomis, 1987). Most recently, Amorim, Glasauer, Corpinot, and Berthoz (1997), Loomis et al. (1992), and Fukusima, Loomis, and Da Silva (1997) have employed two other actions based on the principle of triangulation. In triangulation by pointing, the observer views the target and then attempts to point at the target while walking blindly past it along an oblique path. In triangulation by walking, the observer views the target and then begins walking along an oblique path without vision. On command, the observer turns and faces or walks some distance toward the target. In the former case, the initial and terminal pointing directions are used to triangulate the initially perceived target location; similarly, in the latter case, the terminal heading (or course) of the observer, after the turn, is used, along with the initial target direction, to triangulate the initially perceived target location. Interestingly, the identical analysis was employed by Hill (1979) in connection with jumping spiders of the genus Phidippus during the pursuit of prey. Such spiders do not keep the prey in sight while moving along oblique paths during the pursuit; instead, they pause on occasion to reorient themselves toward the prey and reacquire it visually. Hill used the spider's heading during reorientation toward the prey (which had been removed in the meantime) to determine the spider's estimate of the distance of prey from the initial vantage point. Collett (1982) studied similar updating behavior in toads (Bufo viridis) negotiating opaque detours in the pursuit of prey.

With respect to humans, the general finding in these visually directed tasks is that, with full-cue viewing, there is very little systematic error in the performance of the average observer for targets closer than $15 \mathrm{~m}$ (Corlett, 1992; Elliott, 1986, 1987; Fukusima et al., 1997; Laurent \& Cavallo, 1985; Loomis et al., 1992; Philbeck \& Loomis, 1997; Rieser et al., 1990; Steenhuis \& Goodale, 1988; Thomson, 1983). This absence of systematic error has been interpreted by Loomis and his colleagues as the absence of systematic error in each of the constituent subprocesses: visual perception of the target location, updating of current self-position based on integration of perceived selfvelocity, imaginal updating of the target location based on updated self-position, and, in the case of pointing, executing the pointing response to the updated target location. For similar analyses of the components of visually directed walking, see Böök and Gärling (1981) and Rieser and Rider (1991). Despite the absence of systematic error, however, each of the subprocesses no doubt contributes noise to the overall variability associated with the response.

Contrasting the results obtained with full-cue viewing are results obtained by Philbeck and Loomis (1997) showing that when the cues specifying the egocentric distance of the target are greatly reduced, visually directed walking exhibits the pattern of errors expected from ear- lier research on perceived distance-overshooting of targets closer than $2 \mathrm{~m}$ and undershooting of targets farther than $3 \mathrm{~m}$. In two of their experiments, observers viewed targets under four different viewing conditions varying in the availability of egocentric distance cues. Besides walking to previewed targets without vision, observers also made verbal reports of perceived distance under the same viewing conditions. The pattern of overestimating and underestimating in the reduced-cue conditions was much the same as that for walking, but the actual values of verbal report were generally different from those of walking. However, when walked distance was plotted against verbal report of perceived distance, the two indicators were related by a fixed mapping across the different conditions of visual cue availability, suggesting that the two indicators were controlled by the same internal variable, perceived egocentric distance.

In the present experiment we also investigated visually directed action under some of the conditions of varying cue availability used by Philbeck and Loomis (1997); here, however, we used both visually directed walking and a variant based on the triangulation methods mentioned earlier. The basic procedure is depicted in Figure 2 . Observers viewed a target, closed their eyes, and

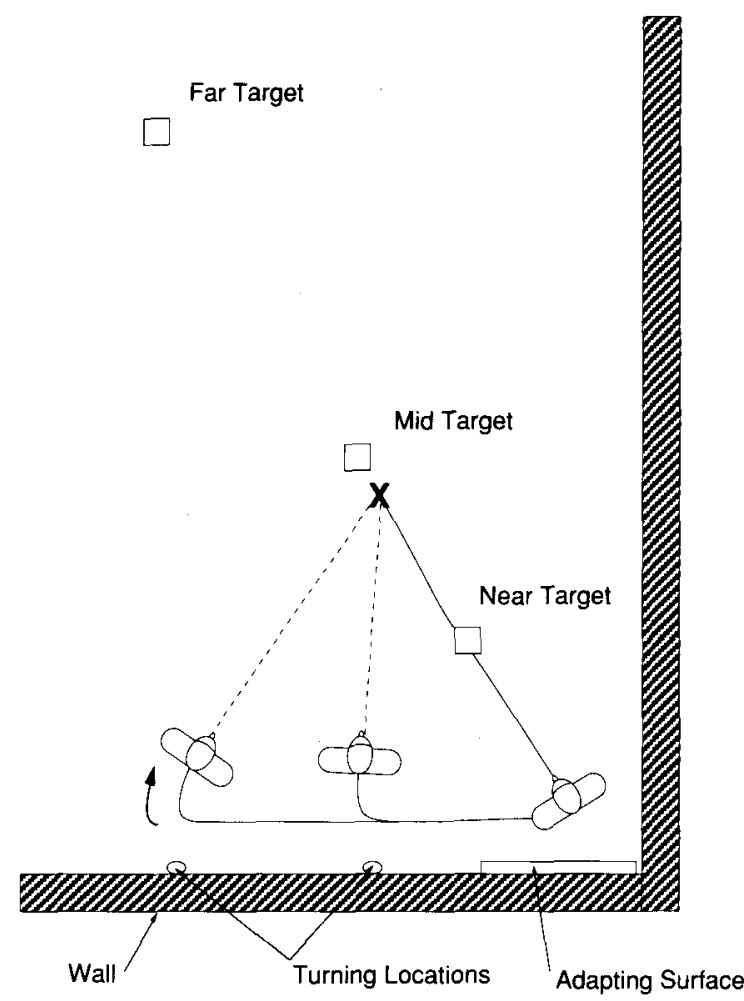

Figure 2. Schematic drawing of a portion of the laboratory space and the target locations, with examples of the three walking paths that might be taken to a particular target. The endpoint of the direct path is denoted with an "X." For clarity, the other two paths are shown uncompleted, but they are projected to show the trajectory endpoints which tend to coincide with that of the direct path. 
then attempted to walk to its location along one of three paths. The observers either walked directly to the target, or walked along a wall oriented obliquely to the target, turned at one of two locations along the wall, and then walked the rest of the way to the target. The observers never knew which path they would be asked to take before closing their eyes. The procedure of indicating the response to be used following the visual exposure means that an observer is unable to preprogram the motor response while visual information is still available; instead, the observer is forced to imaginally update the initially perceived target location during the traverse (see also Thomson, 1983, and Fukusima et al., 1997, for their adoption of a similar procedure for a similar purpose).

In one condition (lights on) of the following experiment, the observers viewed the target in a well-lit environment, a situation that provides abundant information about target location and typically yields performance without systematic error. In the other condition (lights off ), observers viewed only a glowing rectangle in an otherwise dark room, a situation that produces the characteristic pattern of perceptual error mentioned earlier. If perceived location is an invariant controlling action, we expected to see the walking trajectories to each target converge upon the same location. For the lights-on group, we expected this location to be near the physical target location; for the lights-off group, we expected this point to be nearer or farther than the target location according to the aforementioned pattern of perceptual error.

Although the experiment and its predictions derive from our model's postulating that visually directed action relies on three distinct internal representations (visually perceived target location, updated self-position, and updated location of the target), the experiment is not intended as a critical test for distinguishing between the two conceptions of visually based action mentioned at the beginning of this paper. Although the ecological conception avoids reference to any explicit internal representation and consequently would seem unable to predict performance in a task of visually directed action, it is conceivable that there is some basis for making the same predictions that our model does.

\section{METHOD}

\section{Observers}

The observers in the main experiment were 16 adults $(7$ males and 9 females) from the university community who were paid $\$ 8 / \mathrm{h}$ for a single session of about $1.5 \mathrm{~h}$. Their ages ranged from 20 to 31 years, with their mean age being 23.5 years. The second author, age 49 , also participated in a subsidiary experiment; because he performs visually directed tasks very well under full-cue conditions (as demonstrated occasionally in lectures on this line of research), we were interested in his performance in this experiment. All observers had acuity of at least $20 / 20$, corrected if necessary. All were within normal limits of near and far lateral phoria, and had stereoacuity of 25 seconds of arc or better, as measured with the Keystone Orthoscope. Except for the second author, all observers were naive about the purpose of the experiment.

\section{Design}

The main experiment utilized a 3 walking paths (direct; indirect, turn at $1.5 \mathrm{~m}$; indirect, turn at $3.0 \mathrm{~m}) \times 3$ distances $(150,312$, and $600 \mathrm{~cm}) \times 2$ viewing conditions (lights on/lights off) design with 5 or 6 measurements for each combination of factors. The first two factors were varied within observers and the third was varied between groups, with 8 observers per group. (Had we manipulated the latter factor within observers, the observers receiving the lights-on condition first might have used their knowledge of the target locations to correct their responses in the lights-off condition.) We obtained six measurements per combination from the first 4 observers and then switched to five per combination for the remaining observers to shorten the experiment time. In a few cases for a couple of the observers, we obtained four measurements, owing to procedural problems that were not discovered until later. The within-observers presentation order was fully randomized.

In the subsidiary experiment, the second author participated in both conditions of the main experiment, serving in the lights-off condition on one day and then returning 3 days later to serve in the other condition. Other than his participation in both conditions and his involvement in conceptualizing the experiment, the procedures used for him were the same as for the other observers. (Six measurements were taken for each combination of factors.) He was unaware of the particular values used for the target distances.

\section{Stimuli}

In the lights-off viewing condition, the stimuli were luminous rectangles presented straight ahead at eye level with an orientation normal to the line of sight. The apertures used to produce these rectangles were scaled in physical size by photoreduction so that they each subtended a visual angle of $0.86^{\circ}$ (horizontal) $\times 1.34^{\circ}$ (vertical) when seen at the appropriate target distance. Three target distances were used: 150,312 , and $600 \mathrm{~cm}$. The stimuli produced a constant luminance of $0.43 \mathrm{~cd} / \mathrm{m}^{2}$.

In the lights-on condition, the overhead fluorescent lights were illuminated during stimulus presentation, exposing observers to not only the rectangular aperture in the light box, but also the light box itself and the rest of the laboratory. This added many cues not available to the lights-off viewing condition group, such as binocular disparity, the changing angular size of the visible light box across trials, and the changing angular elevation of the stand supporting the light box. Luminance of the rectangular target, as measured from the front of the light box in the lights-on trials, varied with the box's position in the laboratory because of the placement of the overhead lights, and ranged between 5.72 and $11.20 \mathrm{~cd} / \mathrm{m}^{2}$.

In both conditions, observers viewed the stimuli binocularly and with lateral head motion produced by sliding the feet back and forth over a stick taped to the floor. The stick provided tactile information about the ideal amplitude of translation. This procedure resulted in approximately sinusoidal lateral head motion with a peak-to-peak amplitude of about $58 \mathrm{~cm}$ at a frequency of about $0.5 \mathrm{~Hz}$. The exact rate of translation was self-paced by the observer. Our reason for allowing head movement was that we did not wish to stabilize the head and thus decided to use a known amount of head translation. Previous work that we have done suggests that absolute motion parallax with this amount of head movement is a weak egocentric distance cue (Beall, Loomis, Philbeck, \& Fikes, 1995; Philbeck \& Loomis, 1997).

\section{Apparatus}

Laboratory. The experiment was conducted in a laboratory measuring $7.9 \times 11.3 \mathrm{~m}$. There were numerous floor markings which the observer could see before the experiment began. Some of these markings were used for positioning the stimuli, while others were unrelated to target positions and served to minimize the possibility that observers might be biased by seeing a few isolated 
markers. This was unlikely to be a problem, however, because the observer's attention was frequently engaged in other parts of the laboratory space before the experiment began.

To minimize auditory information which might provide cues to the target's location or to the observer's orientation during locomotion, direct sound was attenuated with tight-fitting hearing protectors (Willson CP-365; noise reduction rating: $25 \mathrm{~dB}$ with attenuation of over $37 \mathrm{~dB}$ for frequencies over $1000 \mathrm{~Hz}$ ) worn over both ears. Verbal instructions to the observer were audible by way of a wireless microphone system (Telex AAR-1 and TW-6); the observer wore the FM receiver in a pocket and the earphones beneath the hearing protectors, while the experimenter wore the FM transmitter and clipped the microphone to his shirt. The microphone signal was amplified and delivered to both of the observer's ears, making localization of the amplified sound impossible (a method developed by Rieser, Guth, \& Hill, 1986). This was tested informally by having the observer close his/her eyes and attempt to point continuously at the experimenter as he walked around in the room, on the basis of the sound of his voice. The microphone signal was amplified until pointing responses seemed to the experimenter to be unrelated to his position.

A floodlamp was directed at a piece of white posterboard on the wall behind the observer. Between trials, the lamp was illuminated and the observer viewed the posterboard binocularly. This was intended to maintain a relatively constant state of light adaptation throughout the experiment. This was especially important for the lights-off group, because the luminous rectangles were sufficiently intense to render other parts of the room visible after several minutes of dark adaptation. Thus, the strong adapting light served to eliminate these potential sources of relative distance information by keeping reflections produced by the luminous rectangle well below threshold. The luminance of the illuminated posterboard was about $31 \mathrm{~cd} / \mathrm{m}^{2}$.

Light box. The light box consisted of an electroluminescent panel housed in a wooden frame. The light from the panel was diffused by two pieces of polystyrene to eliminate visible grain in the stimulus. Cards with apertures of different sizes could be slid in front of the diffusers and removed easily. The light box was mounted on a camera tripod and was adjusted to the observer's eye height. The placement of the tripod and light box was determined by positioning the legs of the tripod so that they were in register with the appropriate floor markers.

Position sensing system. The observer's position in the workspace was sensed by a video tracking system that has been described elsewhere (Loomis, Hebert, \& Cicinelli, 1990). Briefly, each of two video cameras mounted in the corners of the laboratory registers the angular position of a flashlight bulb worn by the observer on a headband. Image processing hardware and software then computes the location of the light source by triangulation. This system recorded the observer's position at $10 \mathrm{~Hz}$ with an absolute accuracy of about $5 \mathrm{~cm}$.

\section{Procedure}

Instruction and training phase. Preliminary equipment adjustments and vision tests were conducted in the laboratory in which the experiment took place. Written instructions asked the observers to walk quickly and decisively to where the target was, stopping when they felt their eyes were where the target had been observed. After reading the instructions, the observers were given three practice trials to familiarize them with the procedure. These practice trials were conducted with the room lights on for both the lights-on and the lights-off groups, but without error feedback. The experimenter served as the target stimulus during the practice trials, standing approximately $1.5-2.5 \mathrm{~m}$ from the observer. The observer practiced using each of the three response paths (direct, indirect/turn at $1.5 \mathrm{~m}$, and indirect/turn at $3 \mathrm{~m}$ ) once.
Experimental phase, lights-off group. The observer viewed the adapting surface binocularly while the experimenter positioned the stimulus. When ready, the experimenter extinguished the adapting light, making the room dark. The observer closed his/her eyes turned around, and began translating back and forth laterally in the manner described previously. When the observer signaled that she/he was in position, the experimenter illuminated the panel via computer, and a brief tone sounded as a signal for the observer to open the eyes. After $5 \mathrm{sec}$, the computer extinguished the panel and another tone sounded as a signal to close the eyes. At this point, the observer lowered a blindfold over the eyes and illuminated a tracking light mounted on the hearing protectors. The experimenter then apprised the observer of which path to take (walk straight to the target, turn at the near point, turn at the far point), and moved the stimulus out of the way. As the observer walked, the computer recorded the walking trajectory. In the direct walking trials, the observer attempted to walk directly to where the target had been. In the indirect walking trials, the observer turned to the left and walked along the wall, sliding the left hand along a chalk tray mounted on the wall. The two turning locations were identified by two pieces of tape on the chalk tray which served as tactual markers as the observer's hand passed over them. When the observer felt the marker specified by the experimenter just after vision was occluded, the observer turned and attempted to walk the rest of the way to the target. When the observer stopped walking, the experimenter led him/her back to the starting position, following a winding path so as to minimize potential error feedback that might be obtained on the return path. Once back at the origin, the observer raised the blindfold to view the adapting surface.

Experimental phase, lights-on group. The procedure for this group was similar to that for the lights-off group, except that the overhead fluorescent lights were illuminated while the observer viewed the stimulus. The experimenter stood next to the stimulus tripod during the viewing period. After the second tone sounded to signal the observer to close the eyes, the experimenter extinguished the overhead lights and told the observer which path to take. The response was again carried out in darkness, with only the tracking light illuminating the workspace.

The experimenter attempted to make the trials approximately equal in duration. For both groups, each trial, including the observer's response, averaged about $1 \mathrm{~min}$; this interval included a pause of about $5 \mathrm{sec}$ after the stimulus was occluded, until the observer began to walk. Elliott (1987) has found no effect of delays of this duration on visually directed walking performance.

\section{RESULTS}

For purposes of illustration, Figure 3 depicts the trajectory data of Observer J.L. who performed in both the lights-off and lights-on conditions. The six panels represent the three target locations crossed with these two conditions. In each panel are shown the six trajectories along each of the three paths to each target (for clarity, only every other point along the trajectory is plotted). The segments parallel to the abscissa correspond to walking alongside the wall; all other segments constitute the observer's responses to the spatially updated targets. Because there were six trajectories for each path, the degree of spatial overlap is an indication of J.L.'s consistency of responding.

For each observer, the trajectory endpoint was determined for each of the four, five, or six trajectories within any combination of factors by noting the last sampled lo- 

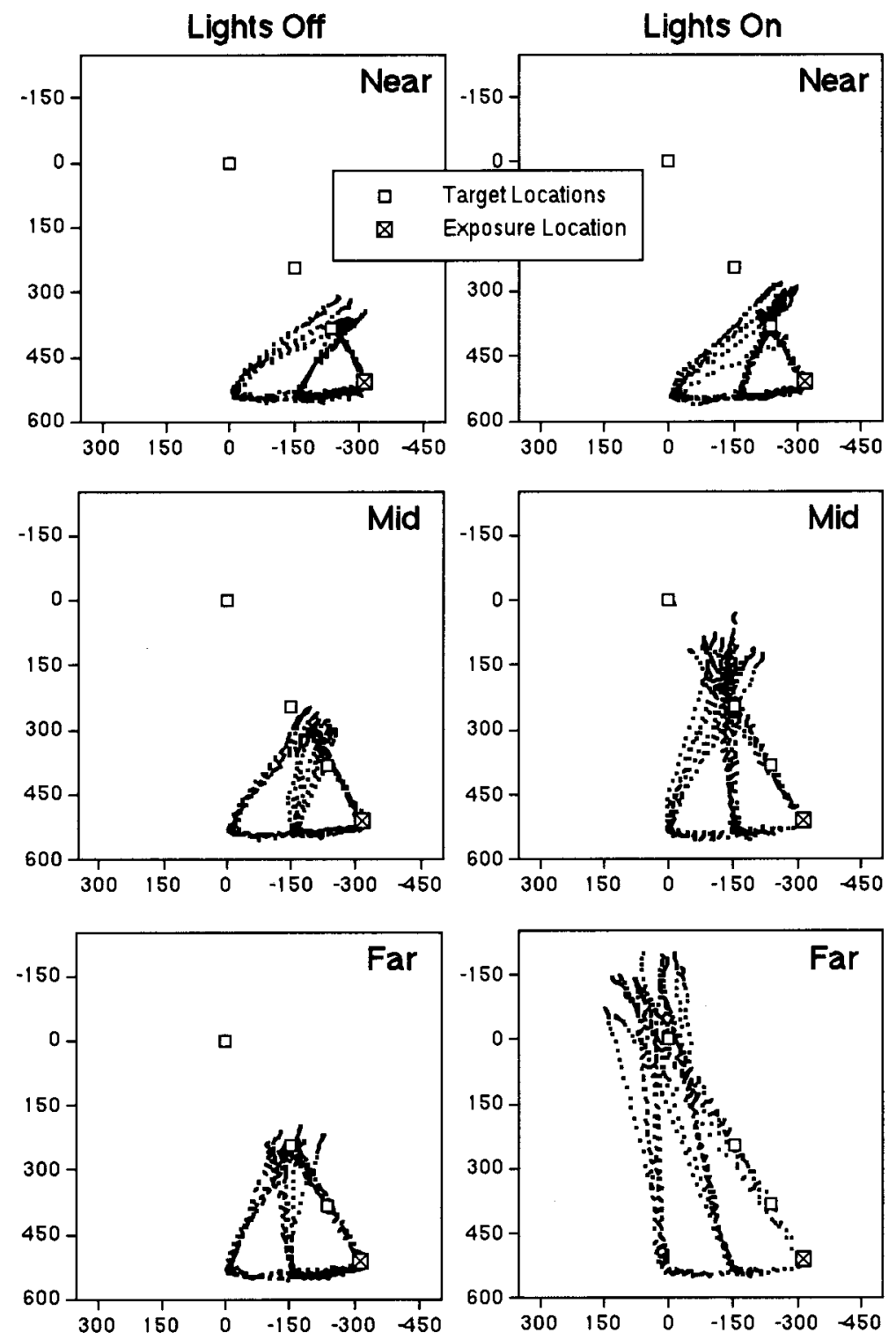

Figure 3. Walking trajectories for Observer J.L., who participated in both the lights-off and the lights-on conditions.

cation in the recorded trajectory. This data was in the form of $x, y$-coordinates, relative to an arbitrary origin (at the far target location). Then, for each observer, target, and path, we computed the centroid of the four (or five or six) trajectory endpoints by averaging the $x$ - and $y$ coordinates separately. Hence, these centroids were the minimal units of the analysis here; that is, we do not evaluate the within-observers variability for a given target or path. We present Figure 4, however, as an example of the range of within-observers variability we found across observers. The left-hand column of figures shows all the endpoints for Observer B.N., whose stopping locations exhibited the greatest spread of any of our observers. The right-hand column shows the endpoints for Observer J.M., whose stopping locations were among the most consis- tent of our observers'. Both B.N. and J.M. happened to be in the lights-off condition.

We assessed the concordance of the observers' stopping locations across the three walking paths by comparing, for each target and each observer, the three centroids, one for each of the three paths. We also computed the grand centroids for each target and path by averaging across observers. These grand centroids are plotted in Figure 5 for both viewing conditions; the error bars along the two orthogonal axes for each grand centroid represent plus and minus one standard error of the mean of the between-observers variability. The three rows of panels show the results for the three target locations (near, mid, and far). The three path types are indicated by straight lines drawn either directly from the starting location to the appropriate grand 


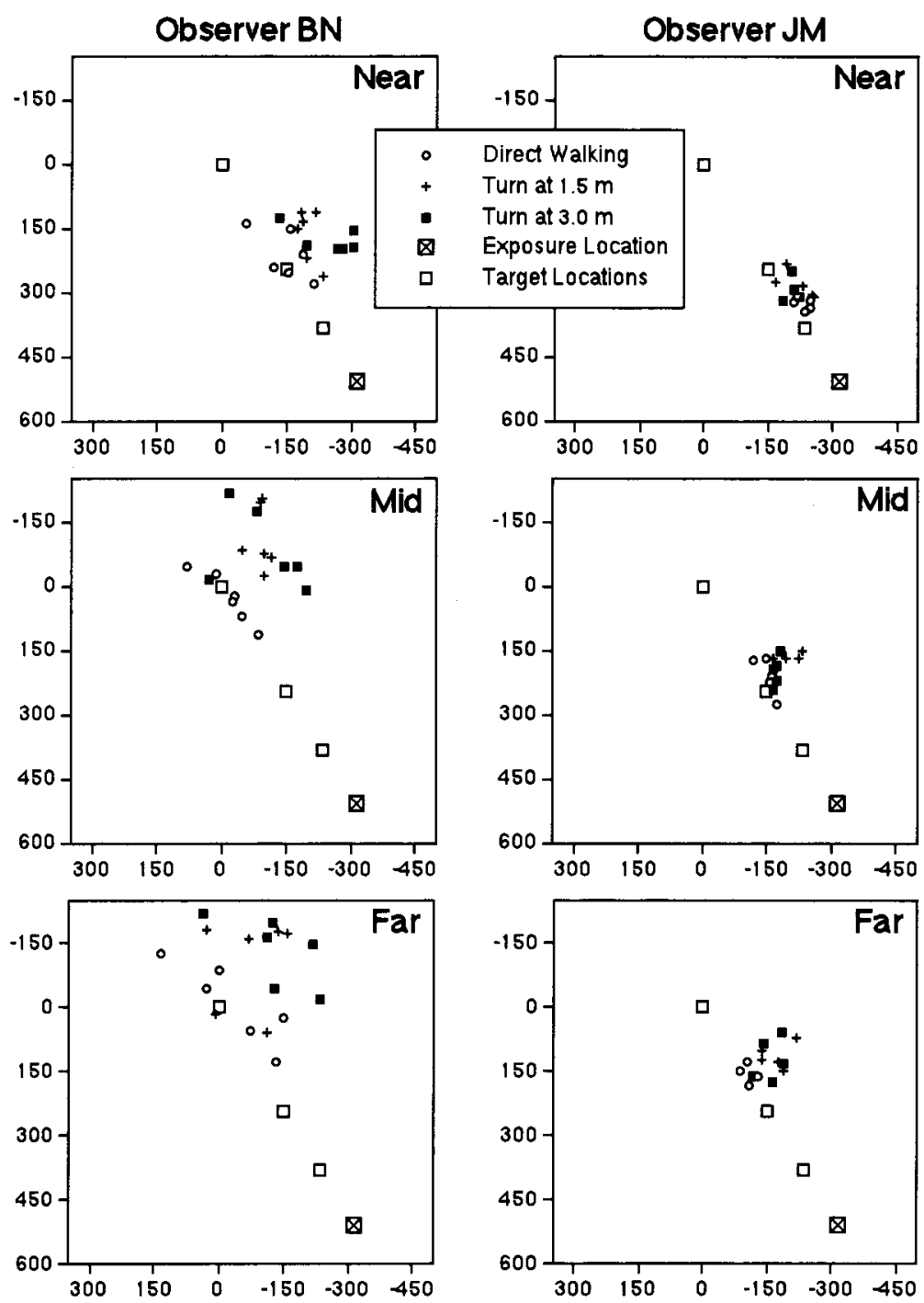

Figure 4. Complete endpoint data for 2 observers in the lights-off group. The endpoints for Observer B.N., whose terminal locations exhibited considerable variability, are shown in the left column of the figure; the endpoints for Observer J.M., whose terminal locations were remarkably consistent, are shown in the right column.

centroid or indirectly between the starting location and the appropriate grand centroids by way of the two turning points. As can be seen, the grand centroids of the stopping locations for each walking path were remarkably close together in both conditions. This indicates that the three walking paths tended to converge upon the same location in space. As we predicted, the stopping locations for the lights-on group were indeed close to the physical target locations, although for the far target the three paths tended to converge upon a location that was about $84 \mathrm{~cm}$ short of the physical target location (for the average observer). We have found some undershooting nearing this magnitude in previous indoor blind walking experiments (Philbeck \& Loomis, 1997). For the lights-off group, the stopping locations for the three paths again tended to converge, but the points of convergence were in poorer agreement with the physical target locations. Responses to the near and middle targets converged on locations somewhat farther than the physical target, whereas responses to the far target converged upon a location somewhat closer than the physical target. This pattern of errors is consistent with the results of previous experiments conducted under reduced-cue conditions, and it has been attributed to perceptual error (e.g., Foley, 1977; Gogel, Loomis, Newman, \& Sharkey, 1985; Gogel \& Tietz, 1973, 1979).

For a statistical analysis of these data, we used the $x$ and $y$-coordinates of the centroid obtained for each target, path, condition, and observer. We conducted a repeated measures multivariate analysis of variance (MANOVA) on these two coordinates, with the lights-on and lightsoff manipulation treated as a between-groups variable and the path type and target distance manipulations treated as within-observers variables. We modeled the data as a bi- 

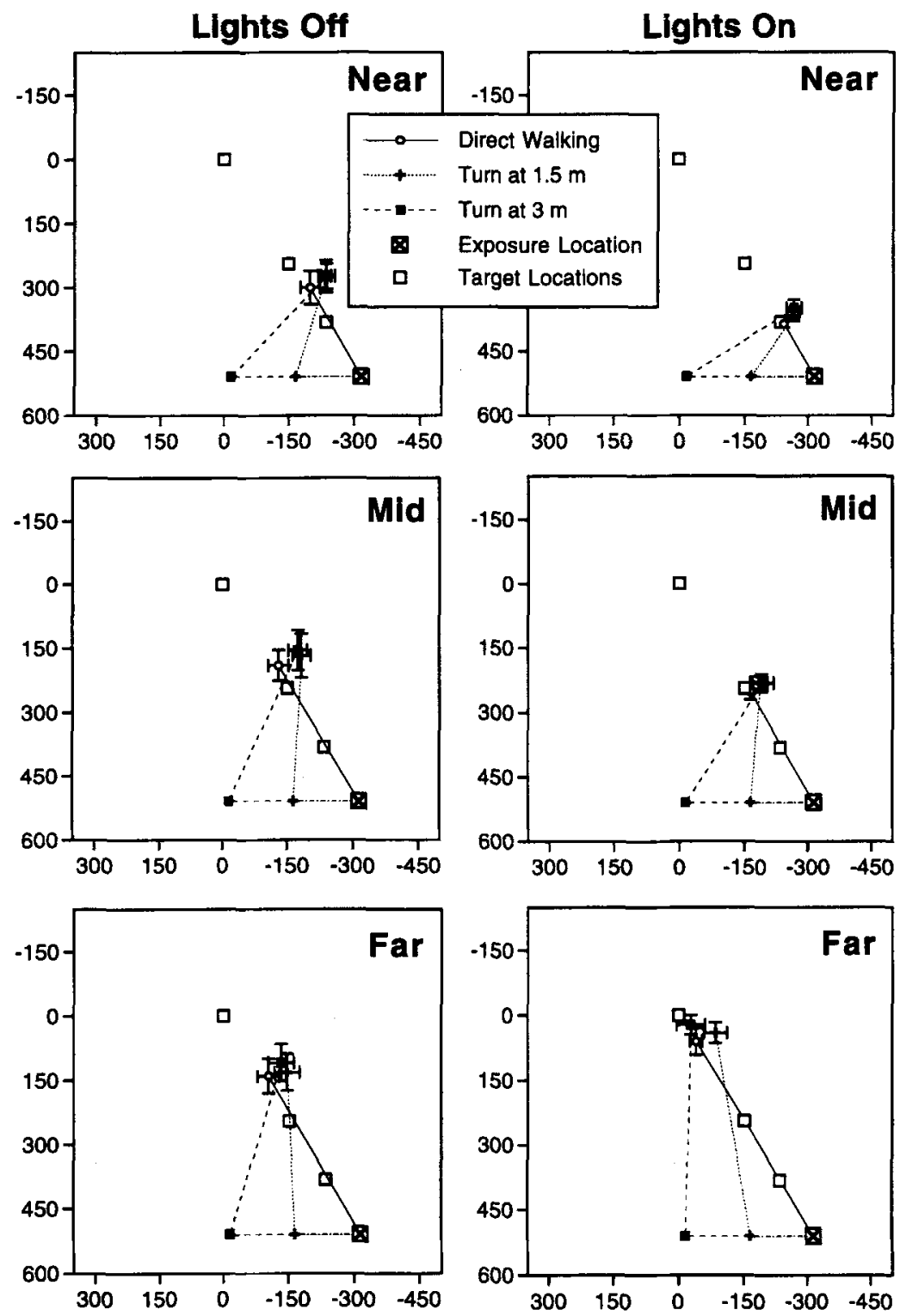

Figure 5. Grand centroids of the endpoints of the three walking paths, averaged across observers. Results for the lights-off and lights-on groups are shown in the left and right panels of the figure, respectively. The units are in centimeters, relative to an arbitrary origin centered on the far target location. The error bars show $\pm 1 S E M$.

variate normal distribution, with the $x$ - and $y$-coordinates as the two dependent variables. When calculating $F$ ratios, we computed Hotelling's $T^{2}$, a statistic that simultaneously tests the $x$ - and $y$-coordinates and takes into account the covariation between these two dependent variables. The analysis did reveal a small but reliable effect of path type $[F(4,11)=8.94, p<.002]$. (The degrees of freedom in the numerator of the $F$ ratios for the withinobservers factors and interactions reflect the sum of the degrees of freedom across the two dependent measures.) This means that, contrary to our expectation, the average stopping points did vary with path; specifically, the indirect trajectories tended to result in some overshooting of the line connecting the observation location with the target locations. However, these differences are small, especially when compared with the distances that observers walked and with the variation in stopping points across target locations. For each condition and target, we computed the mean of the pairwise separations between the three grand centroids for the different paths (Figure 5); when these values were averaged over targets, the grand mean separation was a mere $66 \mathrm{~cm}$ in the dark and $57 \mathrm{~cm}$ in the light.

Second, the MANOVA indicated no main effect of light condition $[F(2,13)=1.07, p>.05]$. Although this might seem contrary to our prediction that observers would walk to different locations in the two light conditions, the most relevant component in the model is the interaction 
between light condition and target distance. This interaction was indeed highly reliable $[F(4,11)=16.77, p<$ $.0001]$. This reflects the tendency in the data for the lightsoff group to exhibit a compressed range of responses relative to the lights-on group. The lack of a main effect of light condition indicates that despite the compression in response range, the marginal means for the two groups were comparable. Finally, there was a main effect of target distance $[F(4,11)=120.56, p<.0001]$. None of the other interactions were significant [path $\times$ light condition, $F(4,11)=0.51$; path $\times$ distance, $F(8,7)=2.74$; and path $\times$ distance $\times$ light condition, $F(8,7)=0.88$, all $p s>.05$ ]

Fukusima et al. (1997) used a triangulation task similar to our indirect walking task. Instead of walking all the way to the target, however, their observers merely turned and walked just part of the way toward the target after walking along a path oblique to it. The authors calculated the perceived target location as the intersection of the initial courses following the turn. In our study, we can compare the intersections of the initial courses with the actual stopping locations. J.L.'s trajectories (Figure 3) illustrate a difference that can arise between the two measures of perceived location. J.L.'s stopping locations in the lights-on condition reflect some overshooting, particularly to the mid and far targets. However, the courses that J.L. followed tended to intersect at points closer to the target location than were the stopping points. To determine whether this was a general finding for the other observers, we computed the average course that each observer followed when leaving the origin or one of the two turning points. We then projected lines from the origin and from the turning points, oriented according to the average courses taken from those points. The intersections of the lines were then determined by solving the set of simultaneous linear equations. These intersections were then subjected to the same MANOVA that was applied to the centroids of the stopping points. This analysis differed little from the previous analysis. Accordingly, we present only the results of the analysis based on the trajectory endpoints. As a way of distilling the results still further, Figure 6 (Observer J.L.) and Figure 7 (other observers) indi-

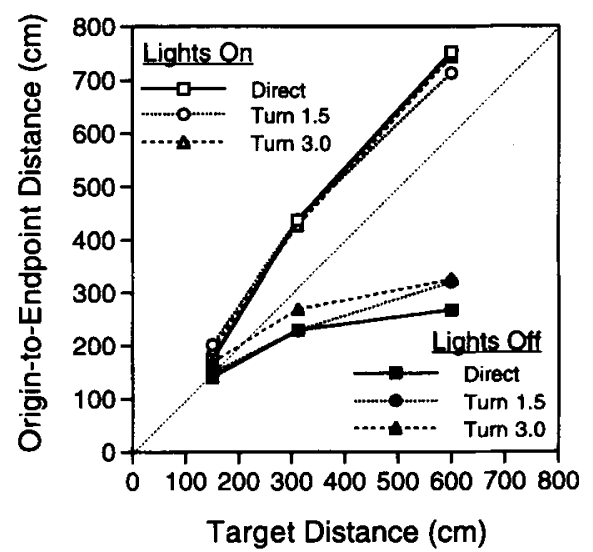

Figure 6. Mean distances from the origin to the stopping locations for Observer J.L., who participated in both conditions.
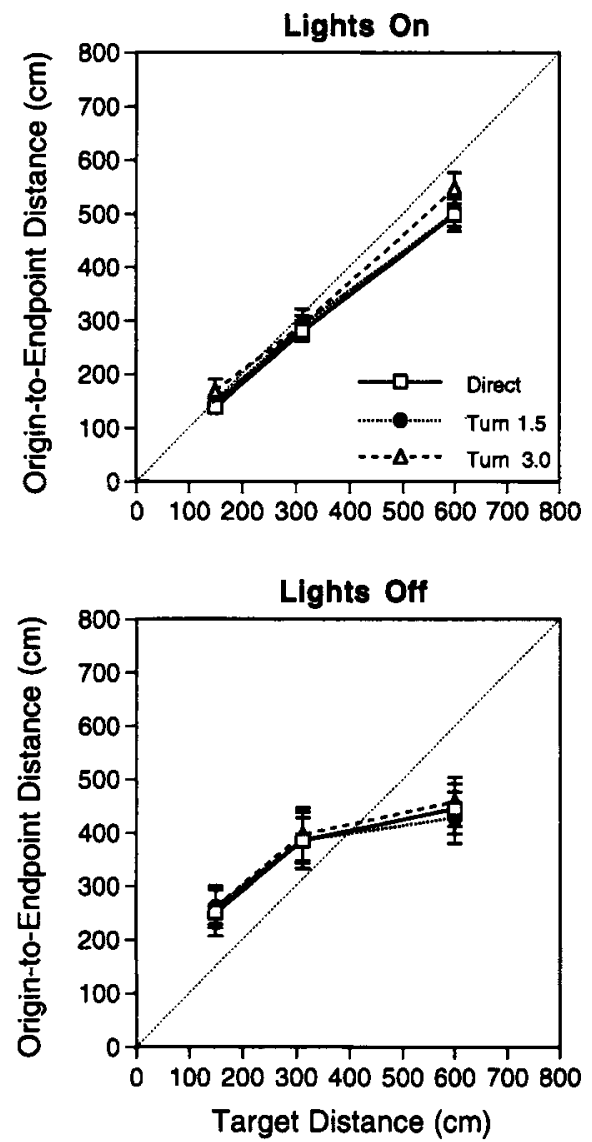

Figure 7. Mean distances from the origin to the stopping locations, averaging across observers, for the lights-on and lights-off conditions. The error bars show $\pm 1 S E M$.

cate the distance between the origin and the centroid of terminal points for different combinations of condition, target, and path. Figure 7 makes clear the overshooting of the near target and the undershooting of the far target in the lights-off condition and more accurate responding in the lights-on condition, albeit with some systematic undershooting to the far target.

In a separate analysis of the distance between the target position and the centroid for each observer, target, and path in the lights-on condition, Observer J.L.'s performance on this measure $(61,140$, and $148 \mathrm{~cm}$ for the near, mid, and far targets) was about midrange within the distributions of this measure for the other observers in that condition $(57,88$, and $189 \mathrm{~cm}$ for near, mid, and far targets). Thus, J.L.'s performance in this experiment was by no means exceptional.

\section{DISCUSSION}

This experiment provides evidence that perceived location is an invariant in the control of locomotion. When the observers attempted to walk without vision to a particular target that had been seen during preview, the walking trajectories tended to terminate at more or less the same location in space whether the observer walked along a 
direct path to the target or along one of two indirect paths. Equally as important, these terminal points varied systematically with the availability of information about target distance. When abundant distance information was available, the different path trajectories converged on locations near the physical target locations. When distance information was restricted, the trajectories converged on locations that were systematically displaced from the physical target locations in a manner consistent with the perceptual errors typically found under restricted viewing conditions. We believe that the simplest interpretation of these results is that observers were directing their open-loop walking to the initially perceived (and subsequently updated) target locations.

Experiments using visually directed walking, triangulation by pointing, and triangulation by walking as indicators of distance perception have indicated that the locations of objects resting on the ground up to at least $15 \mathrm{~m}$ away are correctly perceived when distance information is abundant (Elliott, 1986, 1987; Fukusima et al., 1997; Loomis et al., 1992; Rieser et al., 1990; Steenhuis \& Goodale, 1988; Thomson, 1983). However, when the cues specifying the egocentric distance of the target are greatly restricted, visually directed walking exhibits the errors of perceived distance expected from research using other measures of perceived distance-overshooting of targets closer than $2 \mathrm{~m}$ and undershooting of targets farther than $2 \mathrm{~m}$ (Philbeck \& Loomis, 1997). The present experiment confirms these basic results - the pattern of undershooting and overshooting when distance cues were restricted (lights off) and the more accurate responding when there were plenty of distance cues available (lights on). However, in contrast with previous results, the average response of the 8 observers in the lights-on condition to the far target at $6 \mathrm{~m}$ undershot the physical target location by $84 \mathrm{~cm}$. (Observer J.L., however, exhibited the opposite tendency in the same condition.)

As mentioned in the introduction, we interpret performance in tasks of visually directed action in terms of four component processes: visual perception of the target, updating of self-position, imaginal updating of the target location, and, in the case of pointing, response execution. Because the procedures in our lights-on and lights-off conditions differed only in the availability of distance information during viewing, we conclude that the only direct effect of this manipulation is on the accuracy of the perceptual representation of the target produced during viewing. Thus, we attribute the larger systematic error in the lights-off condition solely to greater perceptual error during target viewing. By implication, we conclude that no systematic error is associated with the other three processes in either viewing condition. (In tasks of visually directed action, the observer actively determines his/her walking trajectory. In contrast, when the observer is passively guided along a path, systematic errors in the updating of self-position do occur; see, e.g., Fujita, Klatzky, Loomis, \& Golledge, 1993; Loomis et al., 1993).
In other research on visually directed action, a process analysis similar to the one above is generally assumed but not always stated (especially, acknowledgment of the initial perceptual stage). One such expression of a similar model was that by Böök and Gärling (1981) in connection with a study conducted under reduced visual cues. In their experiment, observers viewed a dimly illuminated lamp placed on the floor in a darkened room. Observers verbally reported the distance to and azimuth (relative to straight ahead) of the target both before and after a short walk along a path oblique to the target; observers were guided along the path by a moving pattern of dim lamps on the floor. Because the authors were aware that the observers might have misperceived the target location from the origin of locomotion, the authors used the verbal judgments of initial target distance and azimuth to obtain estimates of the observers' perceived target locations (from the origin). The authors then presented the updating data in terms of errors of distance and azimuth between the updated target locations (after the walk) and the "subjective target location" estimated from the initial distance and azimuth judgments. Even with this correction of initial perceptual error, the authors found that the estimates of the updated target did not remain stationary during the traverse but seemed to move along with the observer in a direction parallel to the traverse. They thus concluded that "maintenance of orientation" (their collective term for what we refer to as updating of self-position and the consequent updating of the target location) was far from accurate. However, because our work under full-cue conditions strongly suggests that updating of self-position and of target location are carried out without systematic error, and because these processes ought not to be any different under reduced-cue conditions, we are compelled to seek an alternative interpretation of their data. Giving greater weight to the observers' azimuth judgments, we suspect that observers were in fact initially perceiving the target as closer than their verbal estimates from the origin indicated; evidence of this is the result that once the observer had passed the physical target during the walk, he/she increasingly overestimated the target azimuth the longer he/she had walked. If this interpretation is correct, the egocentric distances of the updated location must also have been quite different from the corresponding verbal estimates. Thus, we believe that the verbal estimates of both initially perceived distance and of the distances to the updated target were very much in error.

Our interpretation of our results is that observers were directing their action toward the initially perceived target. Although people rarely close their eyes while walking or otherwise responding to a previously viewed target, we believe that the process revealed by our experiment is representative of that operating in normal behavior, for observers find the task completely natural. This does not mean, however, that the alternative conception of how vision controls action, discussed in the introduction, is 
without merit. To the contrary, we believe that there are actions that are tightly controlled by continuously available visual stimulation, even by very specific optical variables, like those mentioned in the introduction. This means that the understanding of the visual control of action in general will depend upon the analysis of each of the following: visual stimulation, the perceptual process, and nonperceptual internal representations like those involved in imaginal updating.

Finally, we note the broad applicability of open-loop action as a means of assessing distance perception. Although we have discussed the various actions in the context of vision, they can be employed in order to investigate perception of target location in other sense modalities. Already two studies of auditory distance perception have employed the auditory analogues of visually directed walking (Ashmead, Davis, \& Northington, 1995; Speigle \& Loomis, 1993). In addition, the present authors and their colleagues have used the present method of converging direct and indirect walking to estimate perceived location of auditory sources (Loomis, Klatzky, Philbeck, \& Golledge, 1997). This general approach to measuring perceived location also has the advantage of not depending on language. As such, it can be used to study visual and auditory space perception in nonverbal humans and in nonhuman species. Indeed, as mentioned in the introduction, Hill (1979) anticipated our work on visually directed action with his use of triangulation by walking (our term) as a means of measuring distance perception in a species of jumping spider.

\section{REFERENCES}

Amorim, M.-A., Glasauer, S., Corpinot, K., \& Berthoz, A. (1997). Updating an object's orientation and location during nonvisual navigation: A comparison between two processing modes. Perception \& Psychophysics, 59, 404-418.

Ashmead, D. H., Davis, D. L., \& Northington, A. (1995). Contribution of listeners' approaching motion to auditory distance perception. Journal of Experimental Psychology: Human Perception \& Performance, 21, 239-256.

BaIRD, J. C. (1970). Psychophysical analysis of visual space. Oxford: Pergamon.

BeALL, A. C., \& LoOmIs, J. M. (1996). Visual control of steering without course information. Perception, 25, 481-494.

Beall, A. C., Loomis, J. M., Philbeck, J. W., \& Fikes, T. G. (1995). Absolute motion parallax weakly determines visual scale in real and virtual environments. In Proceedings of the Conference on Human Vision, Visual Processing, and Digital Display (pp. 288-297). Bellingham, WA: Society of Photo-Optical Instrumentation Engineers.

BööK, A., \& GÄRLING, T. (1981). Maintenance of orientation during locomotion in unfamiliar environments. Journal of Experimental Psychology: Human Perception \& Performance, 7, 995-1006.

BRUNSWIK, E. (1956). Perception and the representative design of psychological experiments (2nd ed.). Berkeley: University of California Press.

CALVERT, E. S. (1954). Visual judgments in motion. Journal of the Institute of Navigation, 7, 233-251.

Campbell, D. T. (1966). Pattern matching as an essential in distal knowing. In K. R. Hammond (Ed.), The psychology of Egon Brunswik (pp. 81-106). New York: Holt, Rinehart \& Winston.

Collett, T. S. (1982). Do toads plan routes? A study of the detour behavior of Bufo viridis. Journal of Comparative Physiology A, 146, 261-271
CoRLetT, J. T. (1992). The role of vision in the planning and guidance of locomotion through the environment. In L. Proteau \& D. Elliott (Eds.), Vision and motor control (pp. 375-396). Amsterdam: NorthHolland.

Cutting, J. E., \& Vishton, P. (1995). Perceiving layout and knowing distances: The integration, relative potency, and contextual use of different information about depth. In W. Epstein \& S. Rogers (Eds.), Handbook of perception and cognition: Vol. 5. Perception of space and motion (2nd ed., pp. 69-117). San Diego: Academic Press.

EBy, D. W., \& Loomis, J. M. (1987). A study of visually directed throwing in the presence of multiple distance cues. Perception \& Psychophysics, 41, 308-312.

ElliotT, D. (1986). Continuous visual information may be important after all: A failure to replicate Thomson (1983). Journal of Experimental Psychology: Human Perception \& Performance, 12, 388-391.

ELLIOTT, D. (1987). The influence of walking speed and prior practice on locomotor distance estimation. Journal of Motor Behavior, 19 476-485.

FOLEY, J. M. (1977). Effect of distance information and range on two indices of visually perceived distance. Perception, 6, 449-460.

Foley, J. M. (1980). Binocular distance perception. Psychological Review, 87, 411-434.

Fujita, N., Klatzky, R. L., LoOmis, J. M., \& Golledge, R. G. (1993) The encoding-error model of pathway completion without vision. Geographical Analysis, 25, 295-314.

Fukusima, S. S., Loomis, J. M., \& DA Silva, J. A. (1997). Visual perception of egocentric distance as assessed by triangulation. Journal of Experimental Psychology: Human Perception \& Performance, 23, 86-100.

GiBson, J. J. (1958). Visually controlled locomotion and visual orientation in animals. British Journal of Psychology, 49, 182-194.

GiBSON, J. J. (1979). The ecological approach to visual perception. Boston: Houghton-Mifflin.

Glasauer, S., Amorim, M.-A., Vitte, E., \& Berthoz, A. (1994). Goal-directed linear locomotion in normal and labyrinthinedefective subjects. Experimental Brain Research, 98, 323-335.

GoGEL, W. C. (1984). The role of perceptual interrelations in figural synthesis. In P. Dodwell \& T. Caelli (Eds.), Figural synthesis (pp. 31-82). Hillsdale, NJ: Erlbaum.

GoGEL, W. C. (1990). A theory of phenomenal geometry and its applications. Perception \& Psychophysics, 48, 105-123.

GoGEL, W. C. (1993). The analysis of perceived space. In S. C. Masin (Ed.), Foundations of perceptual theory (pp. 113-182). Amsterdam: Elsevier.

Gogel, W. C., Loomis, J. M., Newman, N. J., \& Sharkey, T. J. (1985). Agreement between indirect measures of perceived distance. Perception \& Psychophysics, 37, 17-27.

GoGEL, W. C., \& TIETZ, J. D. (1973). Absolute motion parallax and the specific distance tendency. Perception \& Psychophysics, 13, 284-292.

GoGEL, W. C., \& TIETZ, J. D. (1979). A comparison of oculomotor and motion parallax cues of egocentric distance. Vision Research, 19, $1161-1170$

HILL, D. E. (1979). Orientation by jumping spiders of the genus Phidippus (Araneae: Salticidae) during the pursuit of prey. Behavioral Ecology and Sociobiology, 5, 301-322.

Laurent, M., \& CaVallo, V. (1985). Role des modalités de prise d'informations visuelles dans un pointage locomoteur [The role of visual input modality in a locomotor pointing task]. L'Année Psychologique, 85, 41-48.

LEE, D. N. (1980). Visuo-motor coordination in space-time. In G. E. Stelmach \& J. Requin (Eds.), Tutorials in motor behavior (pp. 281295). Amsterdam: North-Holland.

Loomis, J. M. (1992). Distal attribution and presence. Presence: Teleoperators \& Virtual Environments, 1, 113-119.

Loomis, J. M., Da Silva, J. A., Fujita, N., \& Fukusima, S. S. (1992) Visual space perception and visually directed action. Journal of Experimental Psychology: Human Perception \& Performance, 18, 906-921.

Loomis, J. M., Da Silva, J. A., Philbeck, J. W., \& Fukusima, S. S. (1996). Visual perception of location and distance. Current Directions in Psychological Science, 5, 72-77. 
Loomis, J. M., Hebert, C., \& Cicinelli, J. G. (1990). Active localization of virtual sounds. Journal of the Acoustical Society of America, 88, $1757-1764$.

Loomis, J. M., Klatzky, R. L., Golledge, R. G., Cicinelli, J. G., Pellegrino, J. W., \& Fry, P. A. (1993). Nonvisual navigation by blind and sighted: Assessment of path integration ability. Journal of Experimental Psychology: General, 122, 73-91.

Loomis, J. M., Klatzky, R. L., Philbeck, J. W., \& Golledge, R. G. (1997). Assessing auditory distance perception using perceptually directed action. Manuscript submitted for publication.

MCBeath, M. K., Shaffer, D. M., \& Kaiser, M. K. (1995). How baseball outfielders determine where to run to catch fly balls. $S c i-$ ence, 268, 569-573.

Norman, J, F., Todd, J. T., Perotti, V. J., \& Tittle, J. S. (1996). The visual perception of three-dimensional length. Journal of Experimental Psychology: Human Perception \& Performance, 22, 173-186.

Philbeck, J. W., \& Loomis, J. M. (1997). A comparison of two indicators of perceived egocentric distance under full-cue and reducedcue conditions. Journal of Experimental Psychology: Human Perception \& Performance, 23, 72-85.

Rieser, J. J., Ashmead, D. H., Talor, C. R., \& Youngeuist, G. A. (1990). Visual perception and the guidance of locomotion without vision to previously seen targets. Perception, 19, 675-689.

Rieser, J. J., GuTh, D. A., \& HiLl, E. W. (1986). Sensitivity to perspective structure while walking without vision. Perception, 15, 173-188.

Rieser, J. J., Pick, H. L., JR., Ashmead, D. H., \& Garing, A. E. (1995). Calibration of human locomotion and models of perceptual-motor organization. Journal of Experimental Psychology: Human Perception \& Performance, 21, 480-497.

RiESER, J. J., \& Rider, E. A. (1991). Young children's spatial orientation with respect to multiple targets when walking without vision. Developmental Psychology, 27, 97-107.
Royden, C. S., Crowell, J. A., \& Banks, M. S. (1994). Estimating heading during eye movements. Vision Research, 34, 3197-3214. SPEIGLE, J. M., \& Loomis, J. M. (1993). Auditory distance perception by translating observers. In Proceedings of IEEE 1993 Symposium on Research Frontiers in Virtual Reality (pp. 92-99). Los Alamitos, CA: Institute of Electrical and Electronics Engineers Computer Society.

Steenhuis, R. E., \& Goodale, M. A. (1988). The effects of time and distance on accuracy of target-directed locomotion: Does an accurate short-term memory for spatial location exist? Journal of Motor Behavior, 20, 399-415.

Thomson, J. A. (1980). How do we use visual information to control locomotion? Trends in Neurosciences, 3, 247-250.

ThOMson, J. A. (1983). Is continuous visual monitoring necessary in visually guided locomotion? Journal of Experimental Psychology: Human Perception \& Performance, 9, 427-443.

TuRVEY, M. T., \& CARELlo, C. (1986). The ecological approach to perceiving-acting: A pictorial essay. Acta Psychologica, 63, 133-155.

WARREN W. H. (1990). The perception-action coupling. In H. Bloch \& B. I. Bertenthal (Eds.), Sensory-motor organizations and development in infancy and early childhood (NATO Advanced Science Institutes Series, D: Behavioural and Social Sciences, Vol. 56, pp. 2337). Dordrecht: Kluwer.

WARREN, W. H., \& HaNnon, D. J. (1990). Eye movements and optical flow. Journal of the Optical Society of America A, 7, 160-169.

YILMAZ, E. H., \& WARREN, W. H. (1995). Visual control of braking: A test of the tau hypothesis. Journal of Experimental Psychology: Human Perception \& Performance, 21, 996-1014.

(Manuscript received February 16, 1996; revision accepted for publication July 14, 1996.) 